\title{
Community Use of Physical and Occupational Therapy After Stroke and Risk of Hospital Readmission
}

\author{
Janet K. Freburger, PT, PhD, ${ }^{a, b}$ Dongmei Li, MS, ${ }^{c}$ Erin P. Fraher, $\mathrm{PhD}^{\mathrm{b}, \mathrm{d}}$
}

From the ${ }^{a}$ Department of Physical Therapy, University of Pittsburgh, Pittsburgh, PA; ${ }^{b}$ Cecil G. Sheps Center for Health Services Research, University of North Carolina, Chapel Hill, NC; ${ }^{C}$ Department of Epidemiology, University of North Carolina, Chapel Hill, NC; and ${ }^{d}$ Department of Family Medicine, University of North Carolina, Chapel Hill, NC.

\begin{abstract}
Objectives: To determine whether receipt of therapy and number and timing of therapy visits decreased hospital readmission risk in stroke survivors discharged home.

Design: Retrospective cohort analysis of Medicare claims (2010-2013).

Setting: Acute care hospital and community.

Participants: Patients hospitalized for stroke who were discharged home and survived the first 30 days $(\mathrm{N}=23,413 ;$ mean age $\pm \mathrm{SD}, 77.6 \pm 7.5 \mathrm{y})$. Interventions: Physical and occupational therapist use in the home and/or outpatient setting in the first 30 days after discharge (any use, number of visits, and days to first visit).

Main Outcome Measures: Hospital readmission 30 to 60 days after discharge. Covariates included demographic characteristics, proxy variables for functional status, hospitalization characteristics, comorbidities, and prior health care use. Multivariate logistic regression analyses were conducted to examine the relation between therapist use and readmission.

Results: During the first 30 days after discharge, $31 \%$ of patients saw a therapist in the home, $11 \%$ saw a therapist in an outpatient setting, and $59 \%$ did not see a therapist. Relative to patients who had no therapist contact, those who saw an outpatient therapist were less likely to be readmitted to the hospital (odds ratio, 0.73; 95\% confidence interval, 0.59-0.90). Although the point estimates did not reach statistical significance, there was some suggestion that the greater the number of therapist visits in the home and the sooner the visits started, the lower the risk of hospital readmission.

Conclusions: After controlling for observable demographic-, clinical-, and health-related differences, we found that individuals who received outpatient therapy in the first 30 days after discharge home after stroke were less likely to be readmitted to the hospital in the subsequent 30 days, relative to those who received no therapy.

Archives of Physical Medicine and Rehabilitation 2018;99:26-34
\end{abstract}

Presented to the Academy Health Conference, June 27, 2016, Boston, MA.

Supported by a cooperative agreement (no. U81HP26495) with the Health Workforce Research Centers, with the National Center for Health Workforce Analysis, Bureau of Health Workforce, Health Resources and Services Administration, and U.S. Department of Health and Human Services; the Pharmacoepidemiology Gillings Innovation Lab for the Population-Based Evaluation of Drug Benefits and Harms in Older US Adults (no. GIL 200811.0010); the Center for Pharmacoepidemiology, Department of Epidemiology, UNC Gillings School of Global Public Health; the CER Strategic Initiative of UNC's Clinical Translational Science Award (no. UL1TR001111); the Cecil G. Sheps Center for Health Services Research, UNC; and the UNC School of Medicine

The information, conclusions, and opinions expressed in this article are those of the authors, and no endorsement by the National Center for Health Workforce Analysis, Bureau of Health Workforce, Health Resources and Services Administration, or U.S. Department of Health and Human Services is intended or should be inferred.

Disclosures: none.
Advances in the acute care of stroke have reduced stroke mortality by approximately $40 \%$ in recent decades, ${ }^{1}$ but the incidence of residual impairments in stroke survivors remains high, making stroke a major cause of long-term disability in adults. ${ }^{2-5}$ Motor impairment is the most common and widely recognized impairment after stroke, ${ }^{5}$ affecting $80 \%$ of stroke survivors to varying degrees. ${ }^{3}$ Poststroke cognitive impairments are also fairly common, affecting up to one-third of survivors. ${ }^{6,7}$ Motor and cognitive impairments after stroke affect quality of life, interfering with daily activities, function, and social roles. These impairments also increase the risk of inactivity, falls, and hospital readmission. ${ }^{8-12}$

Physical and occupational therapists play key roles in rehabilitating stroke survivors with motor and cognitive impairments. ${ }^{5}$ 
Clinical practice guidelines recommend rehabilitation evaluation and treatment as soon as possible after hospital admission, ${ }^{13-17}$ but decreasing lengths of stay shift much of the rehabilitation to postacute settings (ie, inpatient rehabilitation facility [IRF], skilled nursing facility [SNF], the patient's home, the outpatient setting). Stroke survivors with more severe limitations and/or the lack of family support are more likely to be discharged to an IRF or SNF, but most (approximately 60\%) are discharged directly home. ${ }^{18,19}$

Literature on the comparative effectiveness and cost of postacute rehabilitation care for patients with stroke and other diagnoses has primarily focused on care in delivered IRFs and SNFs. ${ }^{19-33}$ Less is known for patients who transition from acute care to the community. Although a few studies have examined the use of postacute care in the home, ${ }^{28,34}$ the use of rehabilitation in outpatient settings after hospitalization has largely been ignored.

Understanding all possible postacute care pathways is important because evidence suggests early contact with a therapist and more intense therapy (eg, greater number of visits/time) may promote better recovery after stroke. ${ }^{14,27,35}$ Physical and occupational therapists also play a role in educating patients about stroke recovery, secondary prevention, safe mobility, and self-management of physical activity and exercise. ${ }^{8}$ Such activities promote better health and well-being of stroke survivors and may decrease the risk of hospital readmission, falls, and other adverse health care events. ${ }^{36-38}$ Understanding postacute care pathways for stroke also has implications for models of care delivery and payment that promote care coordination (eg, Accountable Care Organizations, Patient-Centered Medical Homes) and the sharing of financial risk across settings and/or providers (eg, bundled payment).

We examined the group of stroke survivors discharged directly home after stroke to determine whether receipt of physical therapist and/or occupational therapist care in the first 30 days postdischarge (delivered in the home or outpatient setting), the number of therapist visits in the first 30 days, and the timing of the first therapist visit (ie, days from discharge to start of care) were associated with hospital readmission. We hypothesized that receipt of care, more intense care (ie, greater number of visits), and timelier care would be associated with a decreased risk of readmission.

\section{Methods}

\section{Data sources}

Our primary data source was a $20 \%$ random sample of Medicare claims (2010-2013). We extracted data from the Beneficiary Summary, Medicare Provider and Analysis Review, Home Health, Outpatient, Carrier, and Durable Medical Equipment files. The claims data were merged with Area Health Resource file data to obtain information on socioeconomic factors in the county where the stroke survivor resided.

\section{Study design and cohort}

We used a retrospective, cohort design to identify Medicare beneficiaries admitted to short-term, acute care hospitals for stroke.

\section{List of abbreviations:}

IRF inpatient rehabilitation facility

SNF skilled nursing facility
We established a 6-month baseline period prior to admission to assess comorbidities and health care use; the hospitalization period to assess comorbidities and clinical characteristics and therapist use; and a 30-day exposure period after discharge home to assess the use, number, and timing of in-home and outpatient therapist visits. We then assessed the 30-day period after the exposure period (ie, our follow-up period) to identify hospital readmissions (supplemental fig $\mathrm{S} 1$, available online only at http:// www.archives-pmr.org/). Our motivation for this design was to have an exposure period distinct from the outcome assessment period to improve causal inference.

We limited our sample to Medicare beneficiaries who were $\geq 66$ years at admission (to ensure cases were Medicare eligible during the 6-mo baseline period), survived the hospital stay and were discharged directly home, remained at home and survived the first 30 days after discharge, and were continuously enrolled in Medicare Parts A and B (traditional, fee-for-service). We excluded individuals hospitalized for stroke during the 6-month baseline period. We identified stroke based on primary and secondary International Classification of Diseases-9th Revision-Clinical Modifications discharge diagnoses codes ${ }^{39-43}$ and excluded individuals with transient ischemic attack diagnoses (supplemental table S1).

\section{Exposure, outcome, and covariates}

We created several variables to describe therapist exposure in the 30 days after discharge home. We created a categorical variable indicating whether the patient received care from a physical therapist and/or occupational therapist in the home (home health claims), in the outpatient setting (outpatient and carrier claims), or not at all. For patients who had contact with a physical or occupational therapist, we created variables to indicate the number of therapist visits and time to first visit for both settings (home or outpatient). We identified therapist use based on revenue center codes and Healthcare Common Procedure Coding System/Current Procedural Terminology codes for therapy-related procedures (supplemental appendix S1 and supplemental table S2) using an algorithm developed by RTI International. ${ }^{43}$ We combined physical therapist and occupational therapist visits because of the low number of occupational therapist visits overall.

We created a dichotomous outcome variable indicating whether the patient was hospitalized in a short-term, acute care hospital in the subsequent 30 days after the exposure for any reason (data from MedPAR claims, supplemental table S3). We also created several covariates (ie, control variables) to characterize the sociodemographic characteristics of patients (eg, age, race, dual eligibility), the hospitalization (eg, length of stay, intensive care unit use), and comorbidities during baseline and hospitalization. Specifically, we identified Elixhauser ${ }^{44}$ comorbidities during baseline and hospitalization, stroke-related comorbidities (eg, aphasia, dementia) during hospitalization, and frailty comorbidities during baseline. The latter were developed based on the work of Faurot et al. ${ }^{45}$ Finally, we created variables to indicate baseline health care use. Covariate definitions are shown in supplemental table S3.

\section{Analysis}

We conducted descriptive analyses to identify therapist use in the home and outpatient settings. We then conducted several multivariate logistic regression analyses to examine the association 
between therapist use, visits, and timing and the risk of hospital readmission controlling for all covariates previously described. Analyses were conducted in Stata $14^{\mathrm{a}}$ using the robust SE option and clustering on hospital to account for the nonindependence of measures within hospital. ${ }^{46}$

\section{Effect of therapist use in home or outpatient setting}

We used a propensity score technique with inverse probability of treatment weighting with stabilization and trimming (for stabilized weights $>10)^{45-47}$ to estimate treatment effects. Briefly, for each patient, we calculated the conditional probably (propensity score) of receiving care from a physical therapist and/or occupational therapist (in the home and/or outpatient setting) given our defined covariates (see supplemental table S3). We then generated inverse probability of treatment weights for each patient using the propensity scores (ie, patients who had a higher propensity to receive physical therapy and/or occupational therapy were assigned larger weights). The propensity score essentially balances the characteristics (covariates) of treated and untreated subjects mimicking some aspects of a randomized controlled trial. ${ }^{47}$ Once the weights were calculated, we used them in multivariate logistic regression models to examine the effects of therapist use in the home or outpatient setting (relative to no use) on risk of hospital readmission. We conducted analyses on the overall sample and on the following subgroups to assess heterogeneity of treatment effects: men, women, black, white, dual eligible, ischemic stroke, and hemorrhagic stroke.

\section{Effect of number and timing of visits}

These analyses were limited to individuals who received therapist care in the first 30 days after discharge. Because there was variability in the number and timing of visits by setting and because of differences in case mix for patients seen in the home versus outpatient setting, we conducted analyses separately by setting. We created categorical variables for the total number of therapist visits in the first 30 days after discharge based on the quartile distribution of the data for each setting. Patients who received both home health and outpatient care $(n=291)$ were grouped with the home health setting because these individuals received home health first, most of the visits in the 30-day exposure period were for home health, and the number of subjects overall was small $(1 \%$ of the sample). We also created categorical variables to represent the number of days from discharge to the first therapist visit based on the distribution of the data and with a particular focus on the first 2 weeks of the exposure period (ie, we created more granular categories for the time period from 1 to $14 \mathrm{~d}$ ). Because of smaller sample sizes we did not conduct subgroup analyses for the effects of therapist visits and timing.

Our study protocol was reviewed and approved by the University of North Carolina's Institutional Review Board.

\section{Results}

Our sample consisted of 23,413 patients discharged home after stroke (fig 1). In the first 30 days after discharge, $40.8 \%$ of patients $(n=9546)$ had contact with a physical therapist and/or occupational therapist. Thirty-one percent of the sample had contact with a therapist in their home, $11 \%$ had contact with a therapist in an outpatient setting, and $1 \%$ had contact with therapists in both settings. Figure 2 provides detailed information on therapist use.
For patients who had contact with a therapist in the home, the mean and median number of days to the first visit was 5.2 \pm 4.6 and 4, respectively, and the mean number of visits was $7.5 \pm 4.5$. For patients who received outpatient therapy, the mean and median number of days to the first visit was $10.3 \pm 7.6$ and 8 , with a mean of $5.7 \pm 4.6$ visits. Physical therapist use was much more frequent than occupational therapist use and occupational therapist use was most often in combination with physical therapist use.

Table 1 presents select sample characteristics stratified by therapist use. Patients who received care in the home were older, more likely to be black and dual eligible, and generally had greater comorbid illness and baseline health care use than patients who received care in the outpatient setting. Although patients who received no postacute care generally had lower rates of comorbidities and physical impairments relative to those who received postacute care, in several instances these rates were only slightly lower.

Overall, $6.3 \%$ of the sample was readmitted to the hospital 31 to 60 days after discharge home. The unadjusted rate of readmission was highest for patients who received home health (7.4\%), followed by patients who received no therapy (5.9\%) and patients who received outpatient therapy $(5.0 \%)$.

\section{Effect of therapist use on readmission}

Table 2 presents adjusted odds ratios for the effect of therapist use in the first 30 days postdischarge and readmission in the subsequent 30 days. Patients who received outpatient care in the first 30 days of discharge were less likely to be readmitted in the subsequent 30 days relative to patients who had no contact with a therapist (odds ratio, 0.73; 95\% confidence interval, 0.59-0.90; $P=.003$ ). This effect was present in all subgroups; however, estimates were not statistically significant for the 2 smallest subgroups (ie, black, hemorrhagic stroke). The treatment effect was greatest in the dual eligible subgroup (odds ratio, 0.53; 95\% confidence interval, $0.30-0.95 ; P=.03$ ) Patients who received home health therapy and were a woman or white had a higher risk of hospital readmission (odds ratios, 1.16 and 1.19, respectively) relative to those who received no therapy.

\section{Effect of therapist visits on readmission}

Table 3 presents adjusted odds ratios for the effect of therapist visits and hospital readmission. Although findings were not significant, the effect of receiving 8 to 9 home health visits in the first 30 days (relative to $1-4$ home health visits) on hospital readmission approached significance (odds ratio, 0.78; 95\% confidence interval, $0.58-1.03 ; P=.08$ ). The number of outpatient therapist visits was not associated with hospital readmission.

\section{Effect of therapist timing on readmission}

Table 4 presents adjusted odds ratios for the effect of timing of the first therapist visit and hospital readmission. Although the point estimates for time to first home health visit or first outpatient visit suggest that patients seen in the first 2 weeks after discharge have a decreased risk of readmission, these estimates were imprecise (ie, wide confidence intervals) and did not reach statistical significance. 


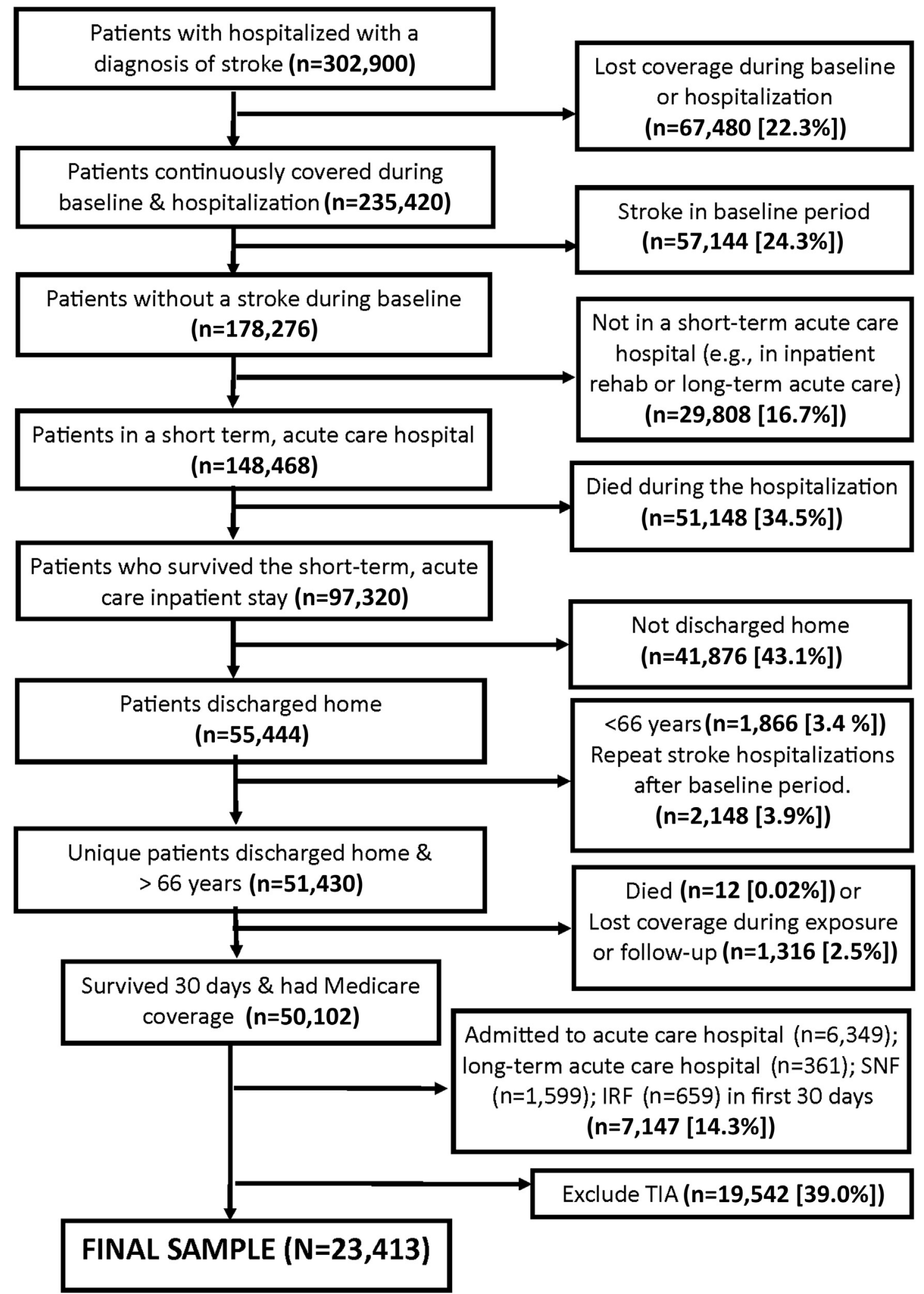

Fig 1 Cohort diagram. 


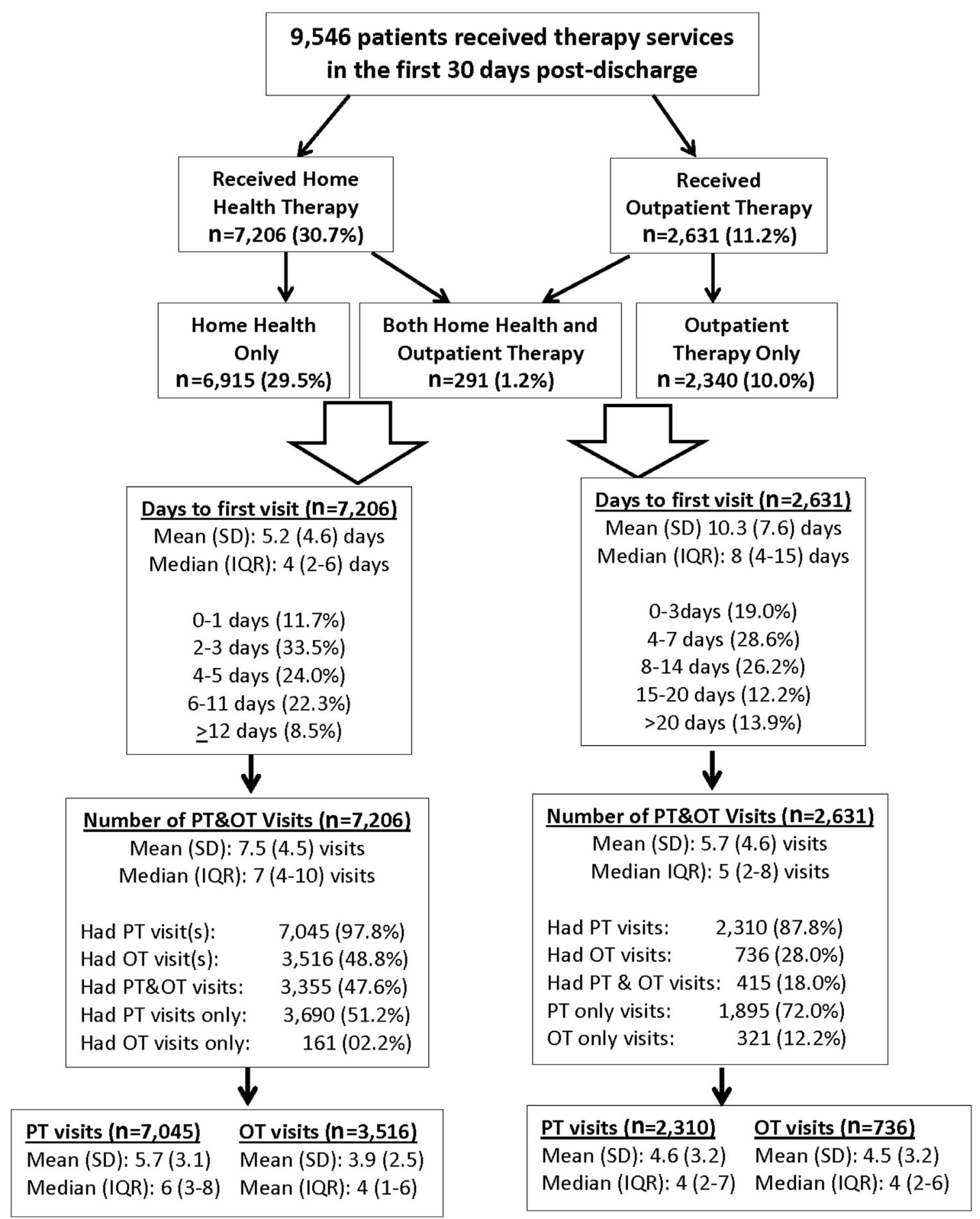

Fig 2 Therapy use during 30-day exposure period. Abbreviations: IQR, interquartile range; 0T, occupational therapist; PT, physical therapist.

\section{Discussion}

We examined the effect of therapist use, therapist visits, and timing of first visit in the first 30 days after discharge home on subsequent hospital readmission in a sample of Medicare beneficiaries hospitalized for stroke. Our most compelling finding was that patients who had contact with an outpatient therapist in the first 30 days after discharge home were less likely to be readmitted to the hospital in the subsequent 30 days, relative to patients who did not have any therapist contact. This effect was consistently observed in the various subgroup analyses and may be because of factors such as improved physical function, engagement with the health care system, and/or patient education on physical activity, falls prevention, stroke risk factors, and so forth. 
Table 1 Sample characteristics by therapist use $(\mathrm{N}=23,413)$

\begin{tabular}{|c|c|c|c|}
\hline \multirow[b]{2}{*}{ Characteristic } & \multicolumn{3}{|c|}{ Postacute Therapist Use } \\
\hline & In Home $(30.8 \%)$ & Outpatient* $(10.0 \%)$ & None $(59.2 \%)$ \\
\hline \multicolumn{4}{|l|}{ Demographic characteristics } \\
\hline Male & 34.0 & 50.0 & 46.0 \\
\hline Mean age $\pm S D, y$ & $79.8 \pm 7.7$ & $75.8 \pm 6.7$ & $76.7 \pm 7.3$ \\
\hline Race White & 76.4 & 87.8 & 82.5 \\
\hline Black & 15.0 & 7.0 & 10.2 \\
\hline Hispanic & 3.8 & 1.6 & 2.7 \\
\hline Other & 4.8 & 3.6 & 4.6 \\
\hline Dual eligibility & 33.6 & 14.1 & 25.3 \\
\hline \multicolumn{4}{|l|}{ Hospitalization characteristics } \\
\hline Stroke Hemorrhagic & 12.4 & 10.5 & 12.0 \\
\hline Ischemic & 87.6 & 89.5 & 88.0 \\
\hline Intensive care unit use & 37.8 & 36.7 & 36.6 \\
\hline Coronary care unit use & 15.6 & 13.2 & 14.5 \\
\hline Mean length of stay \pm SD (d) & $4.6 \pm 4.1$ & $3.3 \pm 2.9$ & $3.4 \pm 2.9$ \\
\hline Received care from PT and/or OT & 69.6 & 91.7 & 89.9 \\
\hline \multicolumn{4}{|l|}{ Stroke-related comorbidities } \\
\hline Aphasia & 11.4 & 10.3 & 10.8 \\
\hline Dysphagia & 5.8 & 3.9 & 2.5 \\
\hline Movement abnormalities & 8.0 & 10.8 & 5.8 \\
\hline Hemiparesis/hemiplegia & 18.2 & 21.9 & 13.4 \\
\hline Fall & 1.1 & 0.6 & 0.5 \\
\hline \multicolumn{4}{|l|}{ Elixhauser comorbidities } \\
\hline \multicolumn{4}{|l|}{ Comorbidity count } \\
\hline $0-1$ & 7.2 & 12.2 & 11.9 \\
\hline $2-4$ & 34.2 & 41.2 & 38.9 \\
\hline $5-7$ & 30.8 & 26.6 & 26.6 \\
\hline $8-10$ & 19.6 & 12.2 & 13.2 \\
\hline$>10$ & 18.3 & 7.8 & 9.5 \\
\hline Paralysis & 18.3 & 15.9 & 8.6 \\
\hline Other neurologic & 53.3 & 34.3 & 37.2 \\
\hline Obesity & 6.4 & 4.4 & 4.7 \\
\hline Depression & 16.6 & 9.7 & 9.4 \\
\hline \multicolumn{4}{|c|}{ Baseline frailty comorbidities and health care use } \\
\hline Use of wheelchair & 3.2 & 0.8 & 1.3 \\
\hline Parkinson disease & 1.3 & 0.7 & 0.6 \\
\hline Weakness & 0.6 & 0.2 & 0.3 \\
\hline Vertigo & 6.8 & 6.8 & 6.7 \\
\hline History of a fall & 8.0 & 5.3 & 3.3 \\
\hline Use of oxygen & 6.6 & 2.3 & 4.0 \\
\hline Use of hospital bed & 2.1 & 0.3 & 0.8 \\
\hline Use of assistive devices & 2.8 & 1.2 & 1.4 \\
\hline$\geq 2$ hospitalizations & 11.1 & 5.5 & 5.4 \\
\hline$\geq 1$ SNF admissions & 4.8 & 1.7 & 1.7 \\
\hline Use of inpatient PT or OT & 16.1 & 9.2 & 7.3 \\
\hline Use of PT or OT in home & 21.4 & 3.8 & 5.5 \\
\hline Use of outpatient PT or OT & 10.8 & 20.3 & 7.8 \\
\hline
\end{tabular}

NOTE. Values are percentages or as otherwise indicated.

Abbreviations: OT, occupational therapist; PT, physical therapist.

* Restricted to patients who saw an outpatient therapist only.

Although we had a large sample size, we were generally underpowered to assess the effects of the number and timing of therapist visits on hospital readmission because of the low prevalence of our outcome and the fact that less than half of our sample had contact with a therapist after discharge home. Future work should use $100 \%$ Medicare claims data. Much more work is also needed in understanding what combinations of postacute care provide the greatest value. Although some work has been done comparing IRF versus SNF care after acute care hospitalizations, even this area lacks strong evidence to guide decisions about the most appropriate site for postacute care. Factors other than need often explain whether patients receive IRF or SNF care. ${ }^{23}$

Only $11 \%$ of our sample had contact with an outpatient therapist. Although therapist use in the home was higher (31\%), more 
Table 2 Adjusted* odds ratios for the effect of therapist use on hospital readmission

\begin{tabular}{|c|c|c|c|c|c|}
\hline \multirow{3}{*}{$\begin{array}{l}\text { Sample } \\
\text { Overall sample }(\mathrm{N}=23,413)\end{array}$} & \multirow{3}{*}{$\begin{array}{l}\text { Therapist Use }^{\dagger} \\
\text { Home health }\end{array}$} & \multicolumn{4}{|c|}{ Hospitalized 31-60d After Discharge } \\
\hline & & \multirow{2}{*}{$\frac{\text { Odds Ratio }}{1.10}$} & \multicolumn{2}{|c|}{$95 \%$ Confidence Interval } & \multirow{2}{*}{$\frac{P}{.13}$} \\
\hline & & & 0.97 & 1.25 & \\
\hline & Outpatient & 0.73 & 0.59 & 0.90 & .003 \\
\hline \multirow[t]{2}{*}{ White $(n=19,003)$} & Home health & 1.16 & 1.01 & 1.33 & .04 \\
\hline & Outpatient & 0.74 & 0.59 & 0.92 & .008 \\
\hline \multirow[t]{2}{*}{ Black $(n=2652)$} & Home health & 0.89 & 0.63 & 1.25 & .50 \\
\hline & Outpatient & 0.67 & 0.30 & 1.49 & .33 \\
\hline \multirow[t]{2}{*}{ Dual eligible $(n=6263)$} & Home health & 0.88 & 0.70 & 1.10 & .26 \\
\hline & Outpatient & 0.53 & 0.30 & 0.95 & .03 \\
\hline \multirow[t]{2}{*}{ Male $(n=9995)$} & Home health & 0.99 & 0.81 & 1.21 & .94 \\
\hline & Outpatient & 0.73 & 0.55 & 0.97 & .03 \\
\hline \multirow[t]{2}{*}{ Female $(n=13,418)$} & Home health & 1.19 & 1.01 & 1.40 & .04 \\
\hline & Outpatient & 0.70 & 0.51 & 0.96 & .03 \\
\hline \multirow[t]{2}{*}{ Ischemic stroke $(n=20,613)$} & Home health & 1.09 & 0.96 & 1.25 & .19 \\
\hline & Outpatient & 0.75 & 0.60 & 0.94 & .01 \\
\hline \multirow[t]{2}{*}{ Hemorrhagic stroke $(n=2800)$} & Home health & 1.15 & 0.84 & 1.57 & .38 \\
\hline & Outpatient & 0.51 & 0.23 & 1.12 & .09 \\
\hline
\end{tabular}

* Adjusted via inverse probability of treatment weights for demographic characteristics, hospitalization characteristics, comorbidities, and baseline health care use.

${ }^{\dagger}$ Referent is no therapist use.

than half of our sample had no therapist contact. This is in contrast with most of our sample having contact with a therapist in the acute care setting (see table 1). These findings suggest there may be underutilization of therapists in the community after stroke considering current evidence that suggests rehabilitation begin as soon as possible and be as intensive as possible ${ }^{5,8,13-15,17}$ and on prevalence estimates of $80 \%$ to $90 \%$ for motor impairment after stroke. $^{2,3,5}$ The low use of outpatient therapists, in particular, suggests a potential area to target as health care systems and insurers move to episode-based models of care delivery and payment.

Current Medicare payment policy for home health and outpatient therapy may be one explanation for our findings regarding greater therapist use in the home versus the outpatient

Table 3 Adjusted* odds ratios for the effect of therapist visits on hospital readmission by setting

\begin{tabular}{|c|c|c|c|c|}
\hline \multirow[b]{2}{*}{ Visit Type } & \multirow[b]{2}{*}{$\begin{array}{l}\text { No. of } \\
\text { Visits }\end{array}$} & \multicolumn{3}{|c|}{$\begin{array}{l}\text { Hospitalized } 31-60 d \\
\text { After Discharge Home }\end{array}$} \\
\hline & & $\begin{array}{l}\text { Odds } \\
\text { Ratio }\end{array}$ & $\begin{array}{l}95 \% \\
\text { Confidence } \\
\text { Interval }\end{array}$ & $P$ \\
\hline \multirow{4}{*}{$\begin{array}{l}\text { Home health } \\
\text { visits }(n=7206)\end{array}$} & $1-4$ & 1.00 & NA & NA \\
\hline & $5-7$ & 0.94 & $0.73-1.20$ & .60 \\
\hline & $8-9$ & 0.78 & $0.58-1.03$ & .08 \\
\hline & $\geq 10$ & 1.08 & $0.84-1.39$ & .55 \\
\hline \multirow{4}{*}{$\begin{array}{l}\text { Outpatient } \\
\text { visits }(n=2340)\end{array}$} & $1-2$ & 1.00 & NA & NA \\
\hline & $3-5$ & 1.06 & $0.60-1.85$ & .84 \\
\hline & $6-7$ & 1.10 & $0.62-1.96$ & .75 \\
\hline & $\geq 8$ & 1.17 & $0.66-2.09$ & .60 \\
\hline
\end{tabular}

Abbreviation: NA, not applicable.

* Adjusted for demographic characteristics, hospitalization characteristics, comorbidities, and baseline health care use. setting. Therapist care in the home, is covered completely under Medicare Part A if a doctor certifies the patient is homebound and in need of therapy. Outpatient therapist care, however, is covered under Medicare Part B and the patient is responsible for $20 \%$ of the costs either through supplemental insurance or paying out-ofpocket. Another potential reason for this finding may be related to the processes in place at patient discharge. Unlike home health, which is sometimes arranged as part of the discharge process, patients are usually just given a written referral for outpatient therapy without assistance in locating a provider in their area. Other potential barriers include transportation issues, lack of family support, scarcity of outpatient therapists, and lack of physician support for therapist use and advocacy for physical activity/exercise after stroke.

As the population ages and advances in medicine continue to improve the acute care of stroke, the prevalence of strokes survivors is likely to increase. Our findings provide some support for better efforts and policies to promote access to and continuity in the use of therapists in the acute to postacute transition after stroke, particularly for patients discharged home. One area to target is educating nurses, physicians, and other providers in the acute and postacute settings about the roles of therapists and the importance of early and continued care after discharge home. Facilitating the transition of patients across rehabilitation settings (eg, home to outpatient) with the objective of maximizing value by sending patients to the least expensive and safest setting to achieve the best possible outcomes is also important. Finally, the health care team needs to recognize and support the ultimate goal of rehabilitation, which is having the patient self-manage their physical activity and exercise to maintain cardiovascular health and general well-being. Needless to say, seamless communication and information exchange among providers in acute and postacute settings are necessary for effective care coordination and continuity for patients who transition from acute to postacute care. Electronic health records and other electronic forms of information exchange (eg, e-care plans) can potentially begin to address 


\begin{tabular}{|c|c|c|c|c|}
\hline \multirow[b]{2}{*}{ Setting } & \multirow[b]{2}{*}{ Days to First Visit } & \multicolumn{3}{|c|}{ Hospitalized 31-60d After Discharge } \\
\hline & & Odds Ratio & 95\% Confidence Interval & $P$ \\
\hline \multirow[t]{3}{*}{ Home health visits $(n=7206)$} & $1-6$ & 0.82 & $0.56-1.19$ & .29 \\
\hline & $7-14$ & 0.85 & $0.56-1.29$ & .45 \\
\hline & $>14$ & 1.00 & NA & NA \\
\hline \multirow[t]{3}{*}{ Outpatient visits $(n=2340)$} & $1-7$ & 0.85 & $0.45-1.58$ & .60 \\
\hline & $8-13$ & 0.88 & $0.55-1.40$ & .59 \\
\hline & $>13$ & 1.00 & NA & NA \\
\hline
\end{tabular}

Abbreviation: NA, not applicable.

* Adjusted for demographic characteristics, hospitalization characteristics, comorbidities, and baseline health care use.

issues around care transitions and continuity of care. As hospitals move toward alternative payment models that include both acute and postacute care, strengthening continuity of therapist care across settings may be particularly useful in preventing downstream health care costs.

\section{Study limitations}

This study has several limitations. First, findings are limited to Medicare beneficiaries discharged home after stroke who survived the first 30 days at home. Second, we did not have direct measures of need for therapy based on therapist/physician assessment, but rather relied on proxy measures available in our data. Related to this limitation is the observational design of our study, which raises the possibility of unmeasured confounding. A third limitation is our measures of therapist utilization were very general (ie, number, timing of visits) and did not reflect the content or patient adherence. Because of sample size issues and the low use of occupational therapists, we chose to combine the physical therapist and occupational therapist data. Future studies should examine the effects of care delivered by each discipline, further explore the content of the therapy sessions, and include the use of other providers (eg, primary care physician, skilled nursing in the home, social worker). Because contact with other providers could also affect readmission risk, understanding the order and timing of all health care provider contacts is important to fully understand predictors of readmission. One strength of our study was that we had distinct exposure and follow-up periods that did not overlap. This design allowed for more confidence in causal inference, but eliminated individuals readmitted in the first 30 days after discharge home (fig 1). Although 30-day readmissions have been the focus of some alternative payment models (eg, Medicare's Readmissions Reduction Program), these models are evolving and including longer periods of risk sharing. In Medicare's Comprehensive Joint Replacement model, hospitals assume risk for their patients for the first 90 days after discharge. A final limitation is that we examined all-cause hospitalization and did not attempt to identify potentially preventable hospitalizations.

\section{Conclusions}

After controlling for observable demographic, clinical, and healthrelated differences, we found that individuals who received outpatient therapy in the first 30 days after discharge home after stroke were less likely to be readmitted to the hospital in the subsequent 30 days, relative to those who received no therapy.

\section{Supplier}

a. Stata 14; StataCorp.

\section{Keywords}

Occupational therapists; Patient readmission; Physical therapists; Rehabilitation; Stroke

\section{Corresponding author}

Janet K. Freburger, PT, PhD, Department of Physical Therapy, School of Health and Rehabilitation Science, Bridgeside Point 1, Ste 201, 100 Technology Dr, Pittsburgh, PA 15219-3130. E-mail address: janet_freburger@pitt.edu.

\section{References}

1. Carandang R, Seshadri S, Beiser A, et al. Trends in incidence, lifetime risk, severity, and 30-day mortality of stroke over the past 50 years. J Am Med Assoc 2006;296:2939-46.

2. Gresham GE, Fitzpatrick TE, Wolf PA, McNamara PM, Kannel WB, Dawber TR. Residual disability in survivors of stroke-the Framingham study. N Eng J Med 1975;293:954-6.

3. Lawrence ES, Coshall C, Dundas R, et al. Estimates of the prevalence of acute stroke impairments and disability in a multiethnic population. Stroke 2001;32:1279-84.

4. Mozaffarian D, Benjamin EJ, Go AS, et al. Heart disease and stroke statistics-2015 update: a report from the American Heart Association. Circulation 2015;131:e29-322.

5. Pollock A, Baer G, Campbell P, et al. Physical rehabilitation approaches for the recovery of function and mobility following stroke. Cochrane Database Sys Rev 2014;(4):CD001920.

6. Mijajlovic MD, Pavlovic A, Brainin M, et al. Post-stroke dementia - a comprehensive review. BMC Med 2017;15:11.

7. Pendlebury ST, Rothwell PM. Prevalence, incidence, and factors associated with pre-stroke and post-stroke dementia: a systematic review and meta-analysis. Lancet Neurol 2009;8:1006-18.

8. Billinger SA, Arena R, Bernhardt J, et al. Physical activity and exercise recommendations for stroke survivors: a statement for healthcare professionals from the American Heart Association/American Stroke Association. Stroke 2014;45:2532-53.

9. Kind AJ, Smith MA, Liou JI, Pandhi N, Frytak JR, Finch MD. The price of bouncing back: one-year mortality and payments for acute stroke patients with 30-day bounce-backs. J Am Ger Soc 2008;56:999-1005. 
10. Mackintosh SF, Hill KD, Dodd KJ, Goldie PA, Culham EG. Balance score and a history of falls in hospital predict recurrent falls in the 6 months following stroke rehabilitation. Arch Phys Med Rehabil 2006; 87:1583-9.

11. Simpson LA, Miller WC, Eng JJ. Effect of stroke on fall rate, location and predictors: a prospective comparison of older adults with and without stroke. PLoS One 2011;6:e19431.

12. Verheyden GS, Weerdesteyn V, Pickering RM, et al. Interventions for preventing falls in people after stroke. Cochrane Database Sys Rev 2013;(5):CD008728.

13. Lindsay P, Bayley M, McDonald A, Graham ID, Warner G, Phillips S. Toward a more effective approach to stroke: Canadian best practice recommendations for stroke care. CMAJ 2008;178:1418-25.

14. Quinn TJ, Paolucci S, Sunnerhagen KS, et al. Evidence-based stroke r-ehabilitation: an expanded guidance document from the european stroke organisation (ESO) guidelines for management of ischaemic stroke and transient ischaemic attack 2008. J Rehabil Med 2009;41: 99-111.

15. European Stroke Organisation (ESO) Executive Committee, ESO Writing Committee. Guidelines for management of ischaemic stroke and transient ischaemic attack 2008. Cerebrovasc Dis 2008;25:457-507.

16. Donohue A, McLaughlin C, Crowe M, Horgan F. Clinical guideline adherence by physiotherapists working in acute stroke care. Ir Med J 2014; 107:287-9.

17. Dworzynski K, Ritchie G, Fenu E, MacDermott K, Playford ED. Rehabilitation after stroke: summary of NICE guidance. BMJ 2013; 346:f3615.

18. Freburger JK, Holmes GM, Ku LJ, Cutchin MP, Heatwole-Shank K, Edwards LJ. Disparities in postacute rehabilitation care for stroke: an analysis of the state inpatient databases. Arch Phys Med Rehabil 2011; 92:1220-9.

19. Prvu Bettger J, McCoy L, Smith EE, Fonarow GC, Schwamm LH, Peterson ED. Contemporary trends and predictors of postacute service use and routine discharge home after stroke. J Am Heart Assoc 2015;4.

20. Tian W. An all-payer view of hospital discharge to postacute care, 2013: statistical brief \#205. Healthcare Cost and Utilization Project (HCUP) statistical briefs. Rockville: Agency for Healthcare Research and Quality; 2013.

21. Ang YH, Chan DK, Heng DM, Shen Q. Patient outcomes and length of stay in a stroke unit offering both acute and rehabilitation services. Med J Aust 2003;178:333-6.

22. Buntin MB, Colla CH, Deb P, Sood N, Escarce JJ. Medicare spending and outcomes after postacute care for stroke and hip fracture. Med Care 2010;48:776-84

23. Buntin MB, Garten AD, Paddock S, Saliba D, Totten M, Escarce JJ. How much is postacute care use affected by its availability? Health Serv Res 2005;40:413-34.

24. Chen CC, Heinemann AW, Granger CV, Linn RT. Functional gains and therapy intensity during subacute rehabilitation: a study of 20 facilities. Arch Phys Med Rehabil 2002;83:1514-23.

25. Dejong G, Horn SD, Smout RJ, Tian W, Putman K, Gassaway J. Joint replacement rehabilitation outcomes on discharge from skilled nursing facilities and inpatient rehabilitation facilities. Arch Phys Med Rehabil 2009;90:1284-96.

26. Deutsch A, Granger CV, Heinemann AW, et al. Poststroke rehabilitation: outcomes and reimbursement of inpatient rehabilitation facilities and subacute rehabilitation programs. Stroke 2006;37:1477-82.

27. Horn SD, DeJong G, Smout RJ, Gassaway J, James R, Conroy B. Stroke rehabilitation patients, practice, and outcomes: is earlier and more aggressive therapy better? Arch Phys Med Rehabil 2005;86(12 Suppl 2):S101-14.

28. Huckfeldt PJ, Mehrotra A, Hussey PS. The relative importance of post-acute care and readmissions for post-discharge spending. Health Serv Res 2016;51:1919-38.

29. Kane RL. Assessing the effectiveness of postacute care rehabilitation. Arch Phys Med Rehabil 2007;88:1500-4.

30. Kane RL, Chen Q, Finch M, Blewett L, Burns R, Moskowitz M. Functional outcomes of posthospital care for stroke and hip fracture patients under Medicare. J Am Gers Soc 1998;46:1525-33.

31. Keith RA, Wilson DB, Gutierrez P. Acute and subacute rehabilitation for stroke: a comparison. Arch Phys Med Rehabil 1995;76:495-500.

32. Ottenbacher KJ, Karmarkar A, Graham JE, et al. Thirty-day hospital readmission following discharge from postacute rehabilitation in feefor-service Medicare patients. J Am Med Assoc 2014;311:604-14.

33. Wodchis WP, Teare GF, Naglie G, et al. Skilled nursing facility rehabilitation and discharge to home after stroke. Arch Phys Med Rehabil 2005;86:442-8.

34. Mahomed NN, Davis AM, Hawker G, et al. Inpatient compared with home-based rehabilitation following primary unilateral total hip or knee replacement: a randomized controlled trial. J Bone Joint Surg 2008;90:1673-80.

35. Lang CE, Lohse KR, Birkenmeier RL. Dose and timing in neurorehabilitation: prescribing motor therapy after stroke. Curr Opinion Neurol 2015;28:549-55.

36. Andrews AW, Li D, Freburger JK. Association of rehabilitation intensity for stroke and risk of hospital readmission. Phys Ther 2015;95:1660-7.

37. Burke JF, Skolarus LE, Adelman EE, Reeves MJ, Brown DL. Influence of hospital-level practices on readmission after ischemic stroke. Neurol 2014;82:2196-204.

38. Eng JJ, Pang MY, Ashe MC. Balance, falls, and bone health: role of exercise in reducing fracture risk after stroke. J Rehabil Res Dev 2008; 45:297-313.

39. Jones SA, Gottesman RF, Shahar E, Wruck L, Rosamond WD. Validity of hospital discharge diagnosis codes for stroke: the Atherosclerosis Risk in Communities Study. Stroke 2014;45:3219-25.

40. Roumie CL, Mitchel E, Gideon PS, Varas-Lorenzo C, Castellsague J, Griffin MR. Validation of ICD-9 codes with a high positive predictive value for incident strokes resulting in hospitalization using Medicaid health data. Pharmacoepidemiol Drug Saf 2008;17:20-6.

41. Spolaore P, Brocco S, Fedeli U, et al. Measuring accuracy of discharge diagnoses for a region-wide surveillance of hospitalized strokes. Stroke 2005;36:1031-4.

42. Thigpen JL, Dillon C, Forster KB, et al. Validity of international classification of disease codes to identify ischemic stroke and intracranial hemorrhage among individuals with associated diagnosis of atrial fibrillation. Circ Cardiovasc Qual Outcomes 2015;8:8-14.

43. Tirschwell DL, Longstreth WT Jr. Validating administrative data in stroke research. Stroke 2002;33:2465-70.

44. Elixhauser A, Steiner C, Harris DR, Coffey RM. Comorbidity measures for use with administrative data. Med Care 1998;36:8-27.

45. Faurot KR, Jonsson Funk M, Pate V, et al. Using claims data to predict dependency in activities of daily living as a proxy for frailty. Pharmacoepidemiol Drug Saf 2015;24:59-66.

46. StataCorp. Stata user's guide release 14. College Station: Stata Press; 2015.

47. Austin PC. An introduction to propensity score methods for reducing the effects of confounding in observational studies. Multivariate Behav Res 2011;46:399-424. 


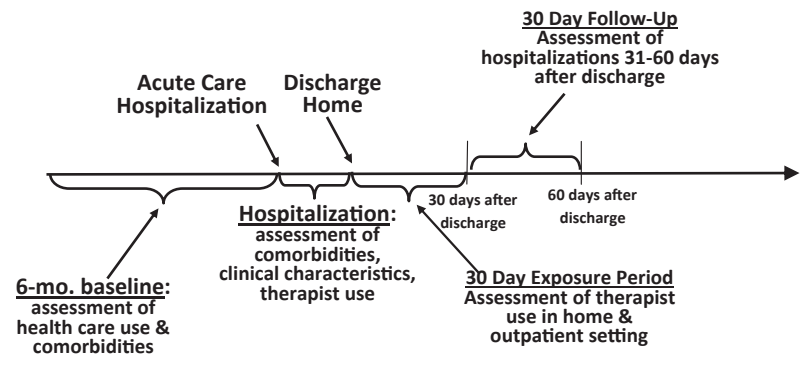

Supplemental fig S1 Study design.
Supplemental Appendix S1 Revenue center codes for therapy

\begin{tabular}{l} 
Revenue Center Codes \\
\hline $0420=$ Physical therapy-general classification \\
$0421=$ Physical therapy-visit charge \\
$0422=$ Physical therapy-hourly charge \\
$0423=$ Physical therapy-group rate \\
$0424=$ Physical therapy-evaluation or reevaluation \\
$0429=$ Physical therapy-other \\
$0430=$ Occupational therapy-general classification \\
$0431=$ Occupational therapy-visit charge \\
$0432=$ Occupational therapy-hourly charge \\
$0433=$ Occupational therapy-group rate \\
$0434=$ 0ccupational therapy-evaluation or reevaluation \\
$0439=$ Occupational therapy-other (may include restorative \\
therapy) \\
$0977=$ Professional fees-physical therapy \\
$0978=$ Professional fees-occupational therapy
\end{tabular}

Supplemental Table S1 ICD-9-CM diagnosis codes to identify stroke and exclude TIA

\begin{tabular}{lll}
\hline ICD-9-CM Code & Description & Type of Stroke \\
\hline 430 & Subarachnoid hemorrhage & Hemorrhagic \\
431 & Intracerebral hemorrhage & Hemorrhagic \\
432 & Other unspecified hemorrhage & Hemorrhagic \\
433.01 & Basilar artery; with cerebral infarction & Ischemic \\
433.11 & Carotid artery; with cerebral infarction & Ischemic \\
433.21 & Vertebral artery; with cerebral infarction & Ischemic \\
433.31 & Multiple and bilateral; with cerebral infarction & Ischemic \\
433.81 & Other specified precerebral artery; with cerebral infarction & Ischemic \\
433.91 & Unspecified precerebral artery; with cerebral infarction & Ischemic \\
434.01 & Cerebral thrombosis; with cerebral infarction & Ischemic \\
434.11 & Cerebral embolism; with cerebral infarction & Ischemic \\
434.91 & Cerebral artery occlusion, unspecified; with cerebral infarction & Ischemic \\
436 & Acute, but ill-defined, cerebrovascular disease & Ischemic \\
437.1 & Other generalized ischemic cerebrovascular disease & Ischemic \\
435 & Transient cerebral ischemia & TIA \\
435.0 & Basilar artery syndrome & TIA \\
435.1 & Vertebral artery syndrome & TIA \\
435.2 & Subclavian steal syndrome & TIA \\
435.3 & Vertebrobasilar artery syndrome & TIA \\
435.8 & Other specified transient cerebral ischemia & TIA \\
435.9 & Unspecified transient cerebral ischemia & TIA \\
\hline
\end{tabular}

Abbreviations: ICD-9-CM, International Classification of Diseases-9th Revision-Clinical Modifications; TIA, transient ischemic attack. 
Supplemental Table S2 Therapy CPT/HCPCS codes*

\begin{tabular}{|c|c|c|c|c|c|}
\hline Code Type & Code & Description & Code Type & Code & Description \\
\hline Proc CPT & 64550 & Apply neurostimulator & Proc CPT & 97032 & Electrical stimulation \\
\hline Proc CPT & 90901 & Biofeedback train, any meth & Proc CPT & 97033 & Electric current therapy \\
\hline Proc CPT & 90911 & Biofeedback peri/uro/rectal & Proc CPT & 97034 & Contrast bath therapy \\
\hline Proc CPT & 92506 & Speech/hearing evaluation & Proc CPT & 97035 & Ultrasound therapy \\
\hline Proc CPT & 92507 & Speech/hearing therapy & Proc CPT & 97036 & Hydrotherapy \\
\hline Proc CPT & 92508 & Speech/hearing therapy & Proc CPT & 97039 & Physical therapy treatment \\
\hline Proc CPT & 92520 & Laryngeal function studies & Proc CPT & 97110 & Therapeutic exercises \\
\hline Proc CPT & 92526 & Oral function therapy & Proc CPT & 97112 & Neuromuscular reeducation \\
\hline Proc CPT & 92597 & Oral speech device eval & Proc CPT & 97113 & Aquatic therapy/exercises \\
\hline Proc CPT & 92605 & Ex for nonspeech device $r x$ & Proc CPT & 97116 & Gait training therapy \\
\hline Proc CPT & 92606 & Nonspeech device service & Proc CPT & 97124 & Massage therapy \\
\hline Proc CPT & 92607 & Ex for speech device $r x, 1 \mathrm{~h}$ & Proc CPT & 97139 & Physical medicine procedure \\
\hline Proc CPT & 92608 & Ex for speech device $r x$ addl & Proc CPT & 97140 & Manual therapy \\
\hline Proc CPT & 92609 & Use of speech device service & Proc CPT & 97150 & Group therapeutic procedures \\
\hline Proc CPT & 92610 & Evaluate swallowing function & Proc CPT & 97530 & Therapeutic activities \\
\hline Proc CPT & 92611 & Motion fluoroscopy/swallow & Proc CPT & 97532 & Cognitive skills development \\
\hline Proc CPT & 92612 & Endoscopy swallow tst (fees) & Proc CPT & 97533 & Sensory integration \\
\hline Proc CPT & 92614 & Laryngoscopic sensory test & Proc CPT & 97535 & Self-care management training \\
\hline Proc CPT & 92616 & Fees $\mathrm{w} /$ laryngeal sense test & Proc CPT & 97537 & Community/work reintegration \\
\hline Proc CPT & 92618 & Ex for nonspeech dev rx add & Proc CPT & 97542 & Wheelchair management training \\
\hline Proc CPT & 95831 & Limb muscle testing, manual & Proc CPT & 97597 & Rmvl devital tis $20 \mathrm{~cm}$ or less \\
\hline Proc CPT & 95832 & Hand muscle testing, manual & Proc CPT & 97598 & Rmvl devital tis addl $20 \mathrm{~cm}$ or less \\
\hline Proc CPT & 95833 & Body muscle testing, manual & Proc CPT & 97602 & Wound(s) care nonselective \\
\hline Proc CPT & 95834 & Body muscle testing, manual & Proc CPT & 97605 & Neg press wound $t x,<50 \mathrm{~cm}$ \\
\hline Proc CPT & 95851 & Range of motion measurements & Proc CPT & 97606 & Neg press wound $\mathrm{tx},>50 \mathrm{~cm}$ \\
\hline Proc CPT & 95852 & Range of motion measurements & Proc CPT & 97750 & Physical performance test \\
\hline Proc CPT & 95992 & Canalith repositioning Proc & Proc CPT & 97755 & Assistive technology assessment \\
\hline Proc CPT & 96105 & Assessment of aphasia & Proc CPT & 97760 & Orthotic management and training \\
\hline Proc CPT & 96110 & Developmental test, lim & Proc CPT & 97761 & Prosthetic training \\
\hline Proc CPT & 96111 & Developmental test, extend & Proc CPT & 97762 & $\mathrm{C} / 0$ for orthotic/prosth use \\
\hline Proc CPT & 96125 & Cognitive test by $\mathrm{HC}$ pro & Proc CPT & 97799 & Physical medicine procedure \\
\hline Proc CPT & 97001 & PT evaluation & Proc CPT & 0019T & Extracorp shock wv tx ms NOS \\
\hline Proc CPT & 97002 & PT reevaluation & Proc CPT & 0183T & Wound ultrasound \\
\hline Proc CPT & 97003 & OT evaluation & Proc HCPCS & G0281 & Electrical stimulation unattend for press \\
\hline Proc CPT & 97004 & OT reevaluation & Proc HCPCS & G0283 & Electrical stimulation other than wound \\
\hline Proc CPT & 97010 & Hot or cold packs therapy & Proc HCPCS & G0329 & Electromagnetic $t x$ for ulcers \\
\hline Proc CPT & 97012 & Mechanical traction therapy & & & \\
\hline Proc CPT & 97016 & Vasopneumatic device therapy & & & \\
\hline Proc CPT & 97018 & Paraffin bath therapy & & & \\
\hline Proc CPT & 97022 & Whirlpool therapy & & & \\
\hline Proc CPT & 97024 & Diathermy (eg, microwave) & & & \\
\hline Proc CPT & 97026 & Infrared therapy & & & \\
\hline Proc CPT & 97028 & Ultraviolet therapy & & & \\
\hline
\end{tabular}

Abbreviations: addl, additional; C/0, care of; CPT, Current Procedural Terminology; dev, device; devital, devitalized; Elec, electric; eval, evaluation; Electromagntic, electromagnetic; Ex, exercise; Extracorp, extracorporeal; HC, healthcare; HCPCS, Healthcare Common Procedure Coding System; lim, limited; meth, method; ms, musculoskeletal system; Neg, negative; NOS, not otherwise specified; OT, occupational therapy; peri, perineal muscles; press, pressure; pro, professional; Proc, procedure; prosth, prosthetic; PT, physical therapy; Rmvl, removal; rx, prescription; stim, stimulation; tis, tissue; tst, test; tx, treatment; uro, urethral sphincter; w/, with; wv, wave.

* For this analysis, speech-related codes and wound therapy codes were excluded. 


\begin{tabular}{|c|c|}
\hline Variable & Definition \\
\hline \multicolumn{2}{|l|}{ Outcome } \\
\hline Hospital readmission & $\begin{array}{l}\text { Hospitalization in a short-term, acute care hospital (excluded long-term acute care, psychiatric, SNF, etc) } \\
\text { for any reason in the first } 30 \mathrm{~d} \text { after the exposure period. }\end{array}$ \\
\hline Sex & Male $=1$, female $=0$ \\
\hline Age & Age at hospital admission, categorized: $66-70,71-75,76-80,81-85,86-90,>90 y$ \\
\hline Race & Categorized as white, black, Hispanic, other (patient's race categorized as other if missing, $<1 \%$ missing) \\
\hline \multicolumn{2}{|l|}{ Hospitalization characteristics } \\
\hline $\begin{array}{l}\text { Patient admitted through emergency } \\
\text { department }\end{array}$ & $1=$ yes, $0=$ no, based on type of admission variable \\
\hline Patient transferred from another hospital & $1=$ yes, $0=$ no, based on source of admission variable \\
\hline Type of stroke & $\begin{array}{l}\text { Ischemic, hemorrhagic, or transient ischemic attack; coded } 0 \text { or } 1 \text { based on ICD-9-CM codes (see } \\
\text { supplemental table S1) }\end{array}$ \\
\hline Physical therapist use & Based on revenue codes for use, coded as 1 if yes, 0 if no (see supplemental appendix S1) \\
\hline Occupational therapist use & Based on revenue codes for use, coded as 1 if yes, 0 if no (see supplemental appendix S1) \\
\hline Speech therapist use & Based on revenue codes for use, coded as 1 if yes, 0 if no \\
\hline \multicolumn{2}{|l|}{ Stroke-related and other relevant comorbidities } \\
\hline Altered consciousness & ICD-9-CM diagnosis codes $780,780.0,780.02,780.03$ \\
\hline Aphasia & ICD-9-CM diagnosis codes $438.1,438.11,784.3,784.6$ \\
\hline Dysphagia & ICD-9-CM diagnosis codes $438.82,787.2,787.20,787.21,787.22,787.23,787.24,787.29$ \\
\hline Aspiration pneumonia & ICD-9-CM diagnosis codes 507, 507.0, 507.1, 507.8 \\
\hline Decubitus & $\begin{array}{l}\text { ICD-9-CM diagnosis codes } 707,707.0,707.1,707.10,707.11,707.12,707.13,707.14,707.15,707.19, \\
\quad 707.2,707.20,707.21,707.22,707.23,707.24,707.25,707.8,707.9\end{array}$ \\
\hline Dementia & ICD-9-CM diagnosis codes 290, 290.1, 290.11, 290.3, 290.4, 290.41, 291.0, 292.81, 293.0, 293.1 \\
\hline Movement abnormalities & ICD-9-CM diagnosis codes 781.0, 781.2, 781.3 \\
\hline Hypertensive heart disease & ICD-9-CM diagnosis codes 402.xx \\
\hline Ischemic heart disease & ICD-9-CM diagnosis codes 410.xx-414.xx \\
\hline
\end{tabular}




Variable
Vascular procedures
Other comorbidities (Elixhauser comorbidities)
Elixhauser Comorbidity Index

Elixhauser Comorbidity Index

Baseline frailty comorbidities

Use of screening tests

Use of wheelchair

Use of other assistive devices

Parkinson disease

Weakness

Vertigo

Falls/difficulty walking

Incontinence

Decubitus

Use of oxygen

Use of hospital bed

Use of ambulance

Nail care
Definition

Data Source

ICD-9-CM diagnosis codes 38.11, 28.12, 00.61-00.65, 17.53, 17.54, 38.01, 38.02, 38.31, 38.32, 38.41, $38.42,38.51,38.52,38.61,38.62,38.81,38.83,39.72,39.75,39.76,39.81-39.89$

29 comorbidity variables (available at: https://www.hcup-us.ahrq.gov/toolssoftware/comorbidity/ comorbidity.jsp)

Created a dichotomous variable for each comorbidity identified during hospitalization and/or during baseline and a categorical count variable $(<2,2-4,5-7,8-10,>10$ comorbidities)

HCPCS/CPT codes: G0009, 90669, 90732, 80061, 82465, 83715, 83716, 83717, 83718, 83719, 83720 $83721,84478,83700,83701,83704, G 0101, G 0202,3014 \mathrm{~F}, 76083,77052,76092,77057,3017 \mathrm{~F}$, G0104, G0105, G0106, G0107, G0120, G0121, G0122, G0328, G0102, G0103, 84153, 84154

ICD-9-CM diagnosis codes: V7644, V771, V7791, V761, V7610, V7611, V7612, V7651

CPT/HCPCS codes: 97542, E0950-E0986, E0988, E0990-E1039, E1050, E1060, E1065, E1066, E1069, E1070, E1083-E1093, E1100, E1110, E1130, E1140, E1150, E1160, E1161，E1170-E1172, E1180, E1190, E1195, E1210-E1213, E1220-E1228, E1240, E1250, E1260, E1270, E1280, E1285, E1290, E1295-E1298，E2201-E2228，E2230，E2231，E2300，E2301，E2310-E2313，E2320-E2331，E2340 -E2343, E2351, E2358-E2377, E2381-E2397, E2399, E2601-E2633, G9156, K0001-K0109, K0114 -K0116, K0195, K0452, K0460, K0461, K0650-K0669, K0733-K0737, K0813-K0816, K0820-K0831, K0835-K0843, K0848-K0864, K0868-K0886, K0890, K0891, K0898, L3964, L3965, L3966

ICD-9-CM diagnosis codes: V463, V538

HCPCS: A4635-A4637, E0100, E0105, E0110-E0114, E0116-E0118, E0130, E0135, E0140-E0149, E0153-E0159, E0163-E0172, E0175, E0240-E0248, K0457-K0459, L0978

ICD-9-CM diagnosis codes: $332,3320,3321$

ICD-9-CM diagnosis codes: 7282, 7283, 7287, 7993, V4984

ICD-9-CM diagnosis codes: 386, 3860, 38600, 38601, 38602, 38603, 38604, 3861, 38610, 38611, 38612, $38619,3862,43885,7804$

ICD-9-CM diagnosis codes: 7197, 71970, 71975, 71976, 71977, 71978, 71979, 7812, V1588, E880, E8800, E8801, E8809, E8842, E8843, E8844, E8845, E8846, E8859, E888, E8880, E8881, E8888, E8889, E9293 ICD-9-CM diagnosis codes: 5965, 59651, 59652, 59653, 59654, 59655, 59659, 7882, 78820, 78821, 78829, $7883,78830,78831,78832,78833,78834,78835,78836,78837,78838,78839$

ICD-9-CM diagnosis codes: 7070, 7071, 70710, 70711, 70712, 70713, 70714, 70715, 70719, 7072, 70720 70721, 70722, 70723, 70724, 70725, 7078, 7079

HCPCS codes: E0431, E0433, E0434, E0435, E0439, E0441, E0442, E0443, E1390, E1393, K0671 HCPCS codes: E0250, E0251, E0255, E0256, E0260, E0261, E0265, E0266, E0270, E0290, E0291, E0292,

E0293, E0294, E0295, E0296, E0297, E0301, E0302, E0303, E0304, E0316, K0456, K0459, K0550 HCPCS codes: A0426, A0427, A0428, A0429, A0999

HCPCS and CPT codes: 11700, 11701, 11710, 11711, 11719, 11720, 11721, G0127, G0247, M0101
Medicare MedPAR file, outpatient file, home health file, carrier file

Medicare MedPAR file, outpatient file, home health file, carrier file, and durable medical equipment file 


\begin{tabular}{|c|c|c|}
\hline Variable & Definition & Data Source \\
\hline \multicolumn{3}{|l|}{ Baseline health care use } \\
\hline Hospitalization & Number of hospitalizations during baseline period categorized as $0,1,2$ or more. & From MedPAR files \\
\hline SNF admissions & SNF admission during baseline period (yes or no) & From MedPAR files \\
\hline Use of PTs or OTs during baseline & $\begin{array}{l}\text { Indicator for outpatient therapy use, home health therapy use, use in short-stay hospital, use in SNF or } \\
\text { long-term care hospital (see supplemental appendix S1 and supplemental table S2) }\end{array}$ & $\begin{array}{l}\text { From MedPAR, outpatient, carrier, } \\
\text { home health files }\end{array}$ \\
\hline Use of speech therapists during baseline & Indicator for speech therapist use during baseline in an inpatient setting based on revenue center codes & From MedPAR files \\
\hline
\end{tabular}

Abbreviations: CPT, Current Procedural Terminology; HCPCS, Healthcare Common Procedure Coding System; ICD-9-CM, International Classification of Diseases-9th Revision-Clinical Modifications; ICU, intensive care unit; MedPar, Medicare provider and analysis review; 0T, occupational therapist; PT, physical therapist. 